\title{
On the number of limit cycles which appear by perturbation of Hamiltonian two-saddle cycles of planar vector fields
}

\author{
Lubomir Gavrilov \\ Institut de Mathématiques de Toulouse, UMR 5219 \\ Université de Toulouse, 31062 Toulouse, France
}

August 7, 2021

2000 MSC scheme numbers: 34C07, 34C08, 34C05

\begin{abstract}
We find an upper bound to the maximal number of limit cycles, which bifurcate from a hamiltonian two-saddle loop of an analytic vector field, under an analytic deformation.
\end{abstract}




\section{Contents}

1 Introduction 2

2 Description of the result 4

3 The Dulac map 8

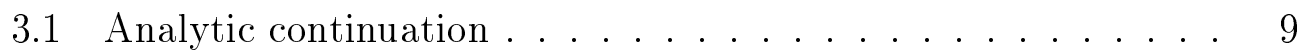

3.2 The Poincaré-Pontryagin integral . . . . . . . . . . . 10

3.3 The zero locus of the imaginary part of the Dulac map . . . 11

4 Cyclicity of two-saddle cycles

A Proof of Theorem 2

B Multi-parameter deformations of Hamiltonian two-saddle loops 21

B.1 Principalization of the Bautin ideal . . . . . . . . . . 21

B.2 Multi-parameter version of Theorem 1 . . . . . . . . . 23

\section{Introduction}

Consider a $N$-parameter analytic family of analytic plane vector fields $X_{\lambda}$, $\lambda \in\left(\mathbb{R}^{N}, 0\right)$, such that $X_{0}$ has a $k$-saddle cycle (a hyperbolic $k$-graphic) $\Gamma_{k}$, as on fig.1. The cyclicity $\operatorname{Cycl}\left(\Gamma_{k}, X_{\lambda}\right)$ of $\Gamma_{k}$ is, roughly speaking, the maximal number of limit cycles of $X_{\lambda}$ which tend to $\Gamma_{k}$ as $\lambda \rightarrow 0$. The first results on the cyclicity of one-saddle connection (also called homoclinic connection) go back to Andronov and Leontovich in 1937 (but they were published only in 1959 [1]). The cyclicity $\operatorname{Cycl}\left(\Gamma_{1}\right)$ has been studied later in full generality by Roussarie [17, 18, see also [19, 12] for an extensive list of references.

The main technical tool of the Roussarie's method is an asymptotic expansion of the Dulac map (transport map near the saddle point)

$$
x \rightarrow d_{\lambda}(x)
$$

in terms of $x^{k}, x^{k} \omega(x, \varepsilon)$ where $\omega(x, \varepsilon)$ is the so called Ecalle-Roussarie compensator

$$
\omega(x, \varepsilon)=\frac{x^{-\varepsilon}-1}{\varepsilon}, \omega(x, 0)=-\ln x, x \in\left(\mathbb{R}^{+}, 0\right)
$$


and $\varepsilon=\varepsilon(\lambda)$ is the trace of the vector field $X_{\lambda}$ at the saddle point. Let $P_{\lambda}$ be the Poincaré first return map, associated to $\Gamma_{1}$ and $X_{\lambda}$. The most delicate case to be studied is when $\Gamma_{1}$ is of infinite co-dimension $\left(P_{0}=i d\right)$. For $\lambda \sim 0$ the map $P_{\lambda}$ is composed by a Dulac map (near the saddle point) and an analytic map (the transport map along the homoclinic orbit). The usual derivation-division algorithm then provides an upper bound for the cyclicity in terms of the number of the coefficients of the asymptotic series of the displacement map $P_{\lambda}-i d$, which vanish as $\lambda=0$. The same method was applied more recently to one-parameter deformations of Hamiltonian twosaddle loops (called also heteroclinic Hamiltonian connections), under the non-generic assumption that one of the separatrices of $\Gamma_{2}$ remains unbroken [4, 3. Recall that a $k$-saddle cycle $\Gamma_{k}$ is said to be Hamiltonian, provided that there is a neighborhood of $\Gamma_{k}$ in which $X_{0}$ allows an analytic first integral with only Morse critical points.

The purpose of the present paper is to extend these results to the case of an arbitrary analytic perturbation of a Hamiltonian two-loop $\Gamma_{2}$, having two hyperbolic equilibrium points.

Our approach is different, as we do not use the asymptotic series of the corresponding Dulac maps $d_{\lambda}^{1}$ and $d_{\lambda}^{2}$, shown on fig.4). Recall that in the one-parameter case $\lambda=\varepsilon \in(\mathbb{R}, 0)$, the displacement function $d_{\varepsilon}^{1}-d_{\varepsilon}^{2}$ can be approximated by an appropriate Abelian integral $I($.$) (or more generally, an$ iterated path integral) depending on a parameter $t$ as follows

$$
d_{\varepsilon}^{1}(t)-d^{2}(t)_{\varepsilon}=\varepsilon^{d} I(t)+\ldots, \varepsilon \sim 0 .
$$

Therefore to count the zeros of $d_{\varepsilon}^{1}-d_{\varepsilon}^{2}$ (corresponding to limit cycles) it is enough to count the zeros of $I($.$) , which can be done by making use of the$ so called "Petrov trick" (based on the argument principle), see [15] and the references given there.

The above considerations hold true at least far from the singular points of the vector field $X_{\lambda}$. As discovered in [4, 3], however, not all limit cycles in a neighborhood of a two-saddle loop can be approximated in such a way. The missing "alien" limit cycles are moreover non-avoidable in generic $N$ parameter deformations $X_{\lambda}$ with $N \geq 4$. For this reason, we apply the argument principle directly to the displacement map $d_{\varepsilon}^{1}()-.d^{2}(.)_{\varepsilon}$ in an appropriate complex domain, in order to obtain an estimate to the number of its complex zeros (corresponding to complex limit cycles).

The main technical result of the paper is Lemma 2, which claims that the zero locus of the imaginary part of the Dulac map is a real analytic curve of 


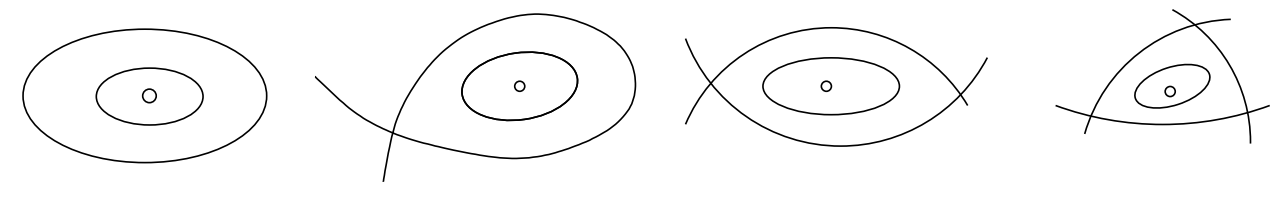

Figure 1: Hamiltonian $k$-saddle cycles

$\mathbb{R}^{2}=\mathbb{C}$ at the origin. This makes possible to investigate the number of the zeros of the imaginary part of $d_{\lambda}^{1}-d_{\lambda}^{2}$ along the zero locus of the imaginary part of $d_{\lambda}^{1}$. Indeed, the intersection numbers of two analytic curves is easily computed. The proof of our main result, Theorem 1, is then completed by making use of the Petrov trick.

In the course of the proof of Theorem 11 we assume, for the sake of simplicity, that our deformations depend on a single small parameter $\varepsilon$. General multi-parameter deformations $\lambda \rightarrow X_{\lambda}$ of $X_{0}$ are then studied along the same lines, as it follows from the Hironaka's desingularization theorem. We explain this in Appendix B.2, see Theorem 4.

Deformations of an arbitrary (possibly non-Hamiltonian) two-loop of infinite co-dimension $\left(P_{\lambda}=i d\right)$ can be studied in a similar way, and will be considered in another paper.

\section{Description of the result}

Let $X_{0}$ be a real plane vector field. Recall that a polycycle of $X_{0}$ is a topological polygon composed of separatrices and singular points. A $k$-saddle cycle of $X_{0}$ (or a hyperbolic $k$-graphic) denoted $\Gamma_{k}$, is a polycycle composed of $k$ distinct saddle-type singular points $p_{1}, p_{2}, \ldots, p_{k}, p_{k+1}=p_{1}$ and separatrices (heteroclinic orbits) connecting $p_{i}$ to $p_{i+1}$. Let $\sigma$ be a segment transversal to the polycycle. The $k$-saddle cycle is said to be Hamiltonian, provided that $X_{0}$ has an analytic first integral $f$ having Morse critical points at $p_{i}$. It follows that $\Gamma_{k}$ bounds an annulus of periodic orbits $\{(x, y): f(x, y)=t\}_{t}$ of $X_{0}$. Thus, a Hamiltonian 0-saddle cycle is simply a center, a Hamiltonian 1-saddle cycle is a homoclinic loop bounding a period annulus, a Hamiltonian 2 -saddle cycle is a double heteroclinic loop bounding a period annulus etc., see fig.1. 
One can find a "tubular neighborhood" $U \subset \mathbb{C}^{2} \cong \mathbb{R}^{4}$ of

$$
\Gamma_{k} \subset\left\{(x, y) \in \mathbb{R}^{2}: f(x, y)=0\right\}
$$

such that

- $\bar{U}$ is compact smooth manifold with a (real three-dimensional) border.

- $f$ is analytic in some neighborhood of $\bar{U}$

- the border $\partial \bar{U}$ is transversal to the complex curves $\left\{(x, y) \in \mathbb{C}^{2}\right.$ : $f(x, y)=t\}$, provided that $|t|$ is sufficiently small.

- the intersection of $U$ with the singular fiber $\left\{(x, y) \in \mathbb{C}^{2}: f(x, y)=0\right\}$ is a union of $k$ Riemann surfaces $D_{i}$, each of them homeomorphic to an open disc. $D_{i}$ intersects transversally $D_{i+1}$ at $p_{i}, i=1 \ldots k$, and $D_{i} \cap D_{j}=\emptyset$ for $|i-j| \neq 1$.

It follows that

$$
f: U \rightarrow \mathbb{C}
$$

defines a locally trivial fibration over a punctured neighborhood of the origin in $\mathbb{C}$, and each fiber

$$
F_{t}=U \cap\left\{(x, y) \in \mathbb{C}^{2}: f(x, y)=t\right\}, t \neq 0
$$

is homeomorphic to a genus one surface with $k$ punctures.

A one-parameter analytic deformation of $X_{0}$ is a a family $X_{\varepsilon}$ of realanalytic plane vector fields, depending analytically on a real parameter $\varepsilon \in$ $(\mathbb{R}, 0)$, and defined in a suitable neighborhood of the $k$-saddle cycle $\Gamma_{k}$. The corresponding foliation $\mathcal{F}_{\varepsilon}$ has an extension in a complex domain denoted by the same letter, and defined by

$$
d f+\varepsilon \omega_{\varepsilon}=0
$$

where $\omega_{\varepsilon}=P(x, y, \varepsilon) d x+Q(x, y, \varepsilon) d y$ is a one-form, and $P, Q$ are realanalytic in $x, y, \varepsilon$ in a neighborhood of $\Gamma_{k}$.

Parameterize the segment $\sigma$ by the "synchronized" local variable $t=\left.f\right|_{\sigma}$ and let $\gamma(t) \subset F_{t}, t>0$ be the continuous family of periodic orbits of $X_{0}$ which tend to the polycycle $\Gamma_{k} \subset F_{0}$ as $t$ tends to 0 . To the family $\{\gamma(t)\}$ we associate the trivial first return map

$$
P_{0}: \sigma \rightarrow \sigma, P_{0}=i d
$$


which allows an analytic continuation for $\varepsilon \neq 0$ to a first return map

$$
\begin{aligned}
P_{\varepsilon}: \sigma & \rightarrow \sigma \\
t & \mapsto P_{\varepsilon}(t)=t+\varepsilon^{d} M_{d}(t)+\ldots
\end{aligned}
$$

The dots above mean a function in $t, \varepsilon$ which, for every fixed $t$ such that $P_{\varepsilon}(t)$, $\varepsilon \sim 0$ is defined, is of the type $0\left(\varepsilon^{d+1}\right)$. The so called Poincaré-Pontryagin function function $M_{d}$ may be explicitly computed, see [16, 5, 6].

More generally, let $\gamma(t) \subset F_{t}$ be any continuous family of closed loops intersecting the cross-section $\sigma$. For $|\varepsilon|$ sufficiently small we define in a similar way the holonomy map

$$
h_{\gamma}^{\varepsilon}: \sigma \rightarrow \sigma
$$

related to the family of loops $\{\gamma(t)\}_{t}$ and the deformed foliation $\mathcal{F}_{\varepsilon}$. By analogy to the Poincaré return map we have

$$
\begin{aligned}
& h_{\gamma}^{\varepsilon}(t)=t+\varepsilon^{d} M_{d}(t)+\ldots \\
& h_{\gamma}^{\varepsilon}: \sigma \rightarrow \sigma \\
& t \mapsto h_{\gamma}^{\varepsilon}(t)=t+\varepsilon^{d} M_{d}(t)+\ldots
\end{aligned}
$$

where the meaning of the dots is as before, and the number $d$ depends on $\{\gamma(t)\}_{t}$ and $\mathcal{F}_{\varepsilon}$.

The holonomy map $h_{\gamma}^{\varepsilon}$ depends on the choice of $\sigma$. In contrast to this, the Poincaré-Pontryagin function $M_{d}$ does not depend on the cross-section $\sigma$, it depends on the free homotopy class of the loop $\gamma(t)$ only. Further, it can be expressed in terms of iterated path integrals of length at most $d$, along suitable meromorphic differential one-forms. It satisfies therefore a linear differential equation which has a Fuchs type singularity at $t=0$, see [7, 9]. Thus, the leading term of $M_{d}$ has the form

$$
t^{p}(\log t)^{q}
$$

where $p$ is an eigenvalue of the indicial equation of the Fuchsian equation related to the regular singular point $t=0$.

Definition 1 We shall call $p$ the characteristic number of the holonomy map $h_{\gamma}^{\varepsilon}$ and denote

$$
\nu\left(h_{\gamma}^{\varepsilon}\right)=p .
$$


In the Hamiltonian case the number $\nu\left(P_{\varepsilon}\right)$ is rational, because the corresponding monodromy operator is quasi-unipotent [10].

To formulate the main result of the paper consider, more specifically, the case $k=2$ ( a double heteroclinic loop). As it follows from [9], the function $M_{d}$ is in fact an Abelian integral and can be written as

$$
M_{d}(t)=\frac{1}{t^{d-1}} \int_{\gamma_{0}(t)} \tilde{\omega}
$$

for suitable analytic one-form $\tilde{\omega}$.

Let $\delta_{1}(t), \delta_{2}(t)$ be two continuous families of closed loops vanishing at the saddle points $p_{1}$ and $p_{2}$. We suppose that orientations of the loops "agree" in the sense that the intersection indices of the homotopy classes of $\delta_{1}(t), \delta_{2}(t)$ with the homotopy class of the periodic orbit $\gamma(t)$ is one and the same.

The cyclicity $\operatorname{Cycl}\left(\Gamma_{2}, \mathcal{F}_{\varepsilon}\right)$ of the 2-saddle cycle $\Gamma_{2}$ with respect to the deformed foliation $\mathcal{F}_{\varepsilon}$ is the maximal number of limit cycles which bifurcate from $\Gamma_{2}$ near $\varepsilon=0$, see [19] for a precise definition. An upper bound for the cyclicity $\operatorname{Cycl}\left(\Gamma_{2}, \mathcal{F}_{\varepsilon}\right)$ is given in terms of the characteristic numbers of the holonomies associated to $\Gamma_{2}$ as follows

\section{Theorem 1}

$$
\operatorname{Cycl}\left(\Gamma_{2}, \mathcal{F}_{\varepsilon}\right) \leq 1+\nu\left(P_{\varepsilon}\right)+\max \left\{\nu\left(h_{\delta_{1}}^{\varepsilon}\right), \nu\left(h_{\delta_{2}}^{\varepsilon}\right)\right\}+\nu\left(h_{\delta_{1}}^{\varepsilon} \circ h_{\delta_{2}}^{\varepsilon}\right) .
$$

It is tempting to conjecture that in general the cyclicity $\operatorname{Cycl}\left(\Gamma_{2}, \mathcal{F}_{\varepsilon}\right)$ is bounded by a similar expression in terms of the characteristic numbers of the holonomies associated to $\Gamma_{k}$. Indeed, in the homoclinic case, $k=1$, by repeating the proof of Theorem 1 one obtains

$$
\operatorname{Cycl}\left(\Gamma_{1}, \mathcal{F}_{\varepsilon}\right) \leq \nu\left(P_{\varepsilon}\right)+\nu\left(h_{\delta_{1}}\right) .
$$

We have, typically

$$
P_{\varepsilon}(t)=t+\varepsilon M_{1}(t)+\ldots
$$

and if

$$
M_{1}(t)=f_{1}(t) \log (t)+f_{2}(t) \not \equiv 0, f_{1}(t)=O\left(t^{p}\right), f_{2}(t)=O\left(t^{q}\right)
$$

for some analytic functions $f_{1}, f_{2}$, then

$$
\operatorname{Cycl}\left(\Gamma_{1}, \mathcal{F}_{\varepsilon}\right) \leq \min \{p, q\}+q
$$


By the Roussarie's theorem [17, Theorem C] the exact upper bound in a real domain in this case is $2 p$ if $p<q$, and $2 q-1$ if $p \geq q$. This suggests that the bound of Theorem 1 can be improved. In fact, the bound (5) holds true for the number of complex limit cycles accumulating on $\Gamma_{2}$ in a suitable neighborhood of it.

Example. Suppose that $d=1$ in $(3)$, that is to say $M_{1}(t)=\int_{\gamma(t)} \omega_{0}$ where $\{\gamma(t)\}_{t>0}$ is the family of real periodic orbits of $\mathcal{F}_{0}=\{d f=0\}$. Then we have

$$
M_{1}(t)=\left(f_{1}(t)+f_{2}(t)\right) \log (t)+f_{3}(t)
$$

where the functions $f_{1}, f_{2}, f_{3}$ are analytic in a neighborhood of $t=0$,

$$
\begin{gathered}
f_{1}(t)=\int_{\delta_{1}(t)} \omega_{0}, f_{2}(t)=\int_{\delta_{2}(t)} \omega_{0} \\
h_{\delta_{1}}(t)=t+\varepsilon f_{1}(t)+\ldots, h_{\delta_{2}}(t)=t+\varepsilon f_{2}(t)+\ldots \\
h_{\delta_{1}} \circ h_{\delta_{2}}(t)=t+\varepsilon\left(f_{1}(t)+f_{2}(t)\right)+\ldots
\end{gathered}
$$

Suppose further that

$$
f_{3}(t)=O\left(t^{p}\right), f_{1}(t)=O\left(t^{p_{1}}\right), f_{2}(t)=O\left(t^{p_{2}}\right), f_{1}(t)+f_{2}(t)=O\left(t^{q}\right) .
$$

Theorem 1 implies that the cyclicity of $\Gamma_{2}$ is bounded by

$$
1+\min \{p, q\}+\max \left\{p_{1}, p_{2}\right\}+q
$$

In the case $p_{1}=p_{2}=q=p$ for instance, this gives

$$
\operatorname{Cycl}\left(\Gamma_{2}, \mathcal{F}_{\varepsilon}\right) \leq 1+3 p
$$

In this situation, and under the strong hypothesis that one of the connections of $\Gamma_{2}$ remains unbroken, it has been proved in [4, Theorem 8] that

$$
\operatorname{Cycl}\left(\Gamma_{2}, \mathcal{F}_{\varepsilon}\right) \leq 2 p-1+\frac{p(p-1)}{2} .
$$

\section{The Dulac map}

Let $\mathcal{F}_{\varepsilon}$ be a real analytic foliation defined by 2 in a neighborhood of a hyperbolic Morse critical point of the function $f$. For all sufficiently small 


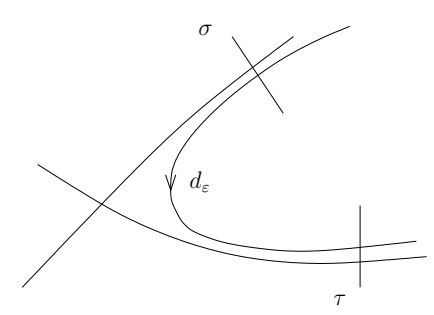

(i)

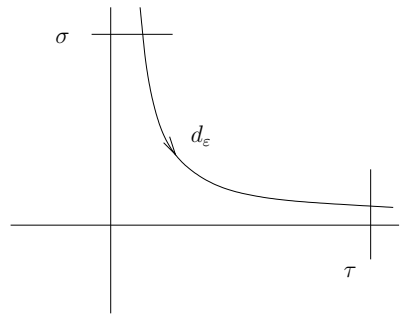

(ii)

Figure 2: The Dulac map

$|\varepsilon|$ the foliation $\mathcal{F}_{\varepsilon}$ has a singular point of saddle type, to which we associate a Dulac map (or the transition map), as on fig.2(i). More precisely, for all sufficiently small $|\varepsilon|$ the foliation $\mathcal{F}_{\varepsilon}$ has two separatrix solutions, which are transversal analytic curves, depending analytically on $\varepsilon$. We may suppose that they are the axes $\{x=0\}$ and $\{y=0\}$ as on fig.2 (ii). Let $\sigma, \tau$ be two complex cross-sections (complex discs) to the two separatrices, parameterized by $z=\left.f\right|_{\sigma}$ and $z=\left.f\right|_{\tau}$. In these coordinates the Dulac map is the germ of analytic map

$$
d_{\varepsilon}:\left(\mathbb{R}_{*}^{+}, 0\right) \rightarrow\left(\mathbb{R}_{*}^{+}, 0\right)
$$

defined as follows: if $z \in \sigma \cap \mathbb{R}_{*}^{+}$then $d_{\varepsilon}(z) \in \tau \cap \mathbb{R}_{*}^{+}$is the intersection with $\tau$ of the orbit $\gamma_{\varepsilon}(z)$ of (2), passing through $z \in \sigma$. This geometric definition of $d_{\varepsilon}$ allows to control to a certain extent its analytic continuation in a complex domain.

\subsection{Analytic continuation}

The Dulac map is analytic and hence allows an analytic continuation on some open subset of the universal covering $\sigma_{\bullet}$ of $\sigma \backslash\{0\}$. The domain of the continuation depends on $\varepsilon$, and obviously $d_{0}(z) \equiv z$.

Let us parameterize the universal covering $\sigma$. by polar coordinates $\rho>$ $0, \varphi \in \mathbb{R}, z=\rho \exp \varphi$.

Theorem 2 There exists $\varepsilon_{0}>0$ and a continuous function

$$
\begin{aligned}
\rho: \mathbb{R} & \rightarrow \mathbb{R}_{*}^{+} \\
\varphi & \mapsto \rho(\varphi)
\end{aligned}
$$


such that the Dulac map allows an analytic continuation in the domain

$$
\left.\{\varepsilon, \rho, \varphi) \in \mathbb{C} \times \sigma_{\bullet}:|\varepsilon|<\varepsilon_{0}, 0<\rho<\rho(\varphi)\right\}
$$

The proof of the above Theorem in the 0-parameter case is well known, and in the multi-parameter case it is the same. For convenience of the reader it will be given in Appendix A. This proof shows even more: the analytic continuation of the Dulac map in the domain (6) can be accomplished in a geometric way as follows.

Let $\left\{\gamma_{0}(z)\right\}_{z}, \gamma_{0}(z) \subset F_{z}$ be a continuous family of loops connecting $\sigma$. to $\tau_{\bullet}$. For $z \in \sigma \cap \mathbb{R}_{*}^{+}$we suppose that $\gamma_{0}(z)$ is the real orbit of $d f=0$ contained in the first quadrant $x \geq 0, y \geq 0$. We note that, although the family $\left\{\gamma_{0}(z)\right\}_{z}$ is not unique, the relative homotopy class of each loop $\gamma_{0}(z)$ is uniquely defined for all $z \in \sigma_{\boldsymbol{\bullet}}$. It follows from the proof of Theorem 2 that $\left\{\gamma_{0}(z)\right\}_{z}$ allows a deformation to a family of paths $\left\{\gamma_{\varepsilon}(z)\right\}_{z}$, connecting $\sigma_{\bullet}$ to $\tau_{\bullet}$, tangent to the leaves of $\mathcal{F}_{\varepsilon}$, and defined for all $\varepsilon, z$ in the domain (6).

\subsection{The Poincaré-Pontryagin integral}

In what follows a crucial role will be played by the integral $\int_{\gamma_{0}} \omega_{0}$, and its generalizations. Namely, let

$$
K \subset\left\{(\rho, \varphi) \in \sigma_{\bullet}: 0<\rho<\rho(\varphi)\right\}
$$

be a compact set, where $\rho(\varphi)$ is as in Theorem 2. As $\int_{\gamma_{\varepsilon}(z)} \omega_{\varepsilon}$ is continuous in $z, \varepsilon$ and

$$
d_{\varepsilon}(z)-z=\int_{\gamma_{\varepsilon}(z)} d f=\varepsilon \int_{\gamma_{\varepsilon}(z)} \omega_{\varepsilon}
$$

then the following Lemma holds

Lemma 1 (Pontryagin[16])

$$
d_{\varepsilon}(z)=z+\varepsilon \int_{\gamma_{0}(z)} \omega_{0}+O\left(\varepsilon^{2}\right)
$$

uniformly in $z \in K$. 
The function $\int_{\gamma_{0}(z)} \omega_{0}$ is the so called Poincaré-Pontryagin integral associated to the deformed foliation $\mathcal{F}_{\varepsilon}$. It follows from the argument principle that, that if $|\varepsilon|$ is sufficiently small, the number of the zeros of $d_{\varepsilon}(z)-z$ in the compact $K$ is bounded by the number of the zeros of the Poincaré-Pontryagin integral $\int_{\gamma_{0}(z)} \omega_{0}$ in $K$ (counted with multiplicity). It might happen, however, that the Poincaré-Pontryagin integral vanishes identically. In all cases there is an integer $d \geq 1$ and an analytic function $M_{d} \neq 0$ in a neighborhood of $K$, such that

$$
d_{\varepsilon}(z)=z+\varepsilon^{d} M_{d}(z)+O\left(\varepsilon^{d+1}\right)
$$

uniformly in $z \in K$, provided that the Dulac map is not the identity map. $M_{d}$ is the so called higher order Poincaré-Pontryagin function and its zeros in $K$ bound as before the number of the zeros of $d_{\varepsilon}(z)-z$. As we already mentioned in section 2, there is an integral representation for $M_{d}$ as an iterated integral of length at most $d$ along $\gamma_{0}(z)$.

Our aim is to obtain a bound for the zeros of $d_{\varepsilon}(z)-z$ in a domain $K$ which is open and connected. Even if the estimate (7), (8) do allow an extension to such a domain $K$, the argument principle can not be directly used. For this purpose we consider rather the imaginary part of the Dulac map.

\subsection{The zero locus of the imaginary part of the Dulac map}

We shall describe the zero locus of the imaginary part of the Dulac map $d_{\varepsilon}$ in an appropriate sector

$$
\begin{aligned}
& \mathcal{H}_{\varepsilon}=\left\{z \in \mathbb{C}: \operatorname{Im} d_{\varepsilon}(z)=0\right\} \cap \mathcal{D} \\
& \mathcal{D}=\left\{0<\rho<\rho(\varphi), 0<\varphi<\frac{3 \pi}{2}\right\} .
\end{aligned}
$$

The surprising fact about $\mathcal{H}_{\varepsilon}$ is that it is a smooth real-analytic plane curve in $\mathcal{D} \subset \mathbb{R}^{2}=\mathbb{C}$. Even better, the curve $\mathcal{H}_{\varepsilon}$ can be conveniently approximated in terms of higher order Poincaré-Pontryagin functions.

The foliation $\mathbb{F}_{0}$ has a first integral defining a fibration with fibers $F_{t}$, see (1). Let $\delta(t) \subset F_{t}$ be a continuous family of closed loops $\delta(t) \subset F_{t}$ vanishing at the saddle point when $t$ tends to 0 . The orientation of $\delta(t)$ is chosen as follows. Let $\gamma_{0}(t)$ be the family of loops defined in the Appendix A. For real 
positive $t$ they coincide with real orbits of $\mathcal{F}_{0}$ connecting $\sigma^{+}$to $\tau^{+}$. Then, the homotopy classes of $\gamma_{0}, \delta$ satisfy

$$
\gamma_{0}\left(t e^{i \pi}\right)-\gamma_{0}\left(t e^{-i \pi}\right)=\delta(t)
$$

Therefore, the exact orientation of $\delta(t)$ can be computed by the PicardLefschetz formula (but we do not need this).

Let $\tau$ be, as before, a cross section to the fiber $F_{0}$, see fig. 2. Consider the holonomy map $h_{\delta}^{\varepsilon}$ associated to the family $\{\delta(t)\}_{t}$ and to the deformed foliation $\mathcal{F}_{\varepsilon}$

$$
\begin{aligned}
h_{\delta}^{\varepsilon: \tau} & \rightarrow \tau \\
z & \mapsto z+\varepsilon^{d} M_{d}(z)+\ldots
\end{aligned}
$$

The anti-holomorphic involution

$$
(x, y) \mapsto(\bar{x}, \bar{y})
$$

induces, for $t \in \mathbb{R}$ an anti-holomorphic involution

$$
F_{t} \rightarrow F_{t}
$$

which on its turn sends the free homotopy class of the loop $\delta(t)$ to the class of $-\delta(t)$. Therefore the function $M_{d}$ is pure imaginary for real values of $t$.

Lemma 2 The zero locus $\mathcal{H}_{\varepsilon}$ of the imaginary part of the Dulac map is a smooth real-analytic curve of $\mathbb{R}^{2}=\mathbb{C}$ of the form

$$
\mathcal{H}_{\varepsilon}=\left\{z=u+i v: v=\frac{\varepsilon^{d}}{2 i} M_{d}(u)+\varepsilon^{d+1} R(u, \varepsilon), u<0\right\} \cap \mathcal{D}
$$

where $R(u, \varepsilon)$ is an analytic function.

The above Lemma is the main technical result of the present paper. The analyticity of the zero locus $\mathcal{H}_{\varepsilon}$ is responsible for the algebraic-like behavior of the Dulac map.

Proof of Lemma 2. Consider the cross-sections (complex discs transversal to the separatrices) $\sigma^{ \pm}, \tau^{ \pm}$as shown on fig. 3 , simultaneously parameterized as before by the restriction $z$ of the first integral $f(x, y)$ on them. The crosssections $\sigma, \tau$ shown on fig. 2 are denoted, from now on, by $\sigma^{+}, \tau^{+}$. Denote

$$
\sigma_{\geq 0}^{+}=\sigma^{+} \cap\{(x, y): f(x, y) \geq 0\}, \sigma_{\leq 0}^{+}=\sigma^{+} \cap\{(x, y): f(x, y) \leq 0\} \text { etc. }
$$




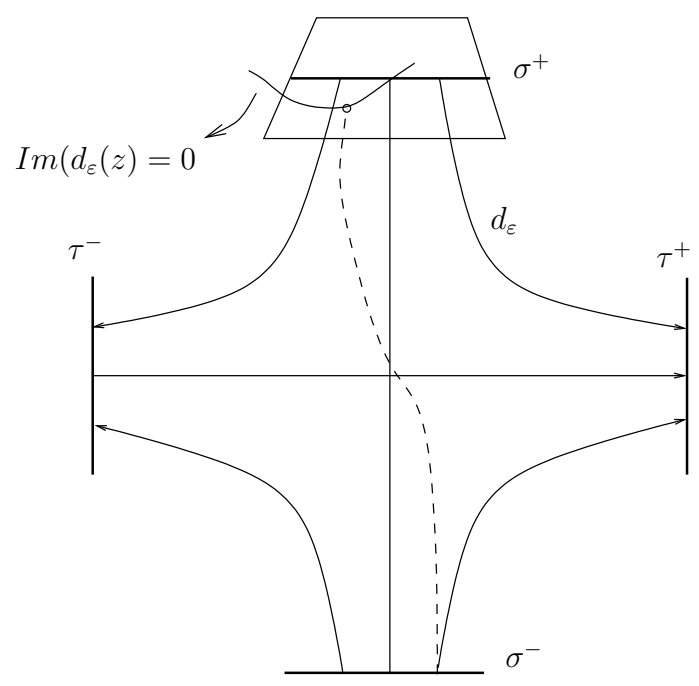

Figure 3: The zero locus of the imaginary part of the Dulac map

Let $\left\{\gamma_{\varepsilon}(z)\right\}_{z}$ be the continuous family of paths, defined in Appendix A. The point $z \in \sigma^{+}$belongs to the zero locus $\mathcal{H}_{\varepsilon}$ if and only if the end of the path $\gamma_{\varepsilon}(z)$ belongs to $\tau_{<0}^{+}$. Therefore, such a path allows a decomposition in a product

$$
\gamma_{\varepsilon}(z)=\alpha_{\varepsilon}(z) \circ \beta_{\varepsilon}(z)
$$

where $\alpha_{\varepsilon}(z)$ is a path connecting $z \in \sigma^{+}$to a point on $\sigma_{<0}^{-}$and $\beta_{\varepsilon}(z)$ is a path connecting the latter point to a point on $\tau_{<0}^{+}$, where $\beta_{\varepsilon}(z) \subset \mathbb{R}^{2}$. It follows that $\mathcal{H}_{\varepsilon}$ is the image of $\sigma_{<0}^{-}$under the holonomy map

$$
h_{\alpha_{0}^{-1}}^{\varepsilon}: \sigma_{<0}^{-} \rightarrow \sigma^{+} .
$$

This already proves that the closure of $\mathcal{H}_{\varepsilon} \subset \mathbb{R}^{2}$ is a smooth analytic curve. Once having said this, it is clear that $\mathcal{H}_{\varepsilon}$ can be conveniently parameterized, which we do next.

To prove $(12)$, let us note that for $t \in \mathbb{R}$ the two ends of the loop $\alpha_{0}(t)$ are real and hence this also holds true for the complex-conjugate loop $\overline{\alpha_{0}(t)}$. The loop $\alpha_{0}(t) \circ \overline{\alpha_{0}^{-1}(t)}$ is therefore closed and is homotopic to $\delta(t) \subset F_{t}$ defined above. 
More explicitely

$$
\begin{aligned}
2 i v & =h_{\alpha_{0}^{-1}}^{\varepsilon}(t)-\overline{h^{\varepsilon}}(t) \\
& =h_{\alpha_{0}^{-1}}^{\varepsilon}(t)-h \frac{\varepsilon}{\alpha_{0}^{-1}}(t) \\
& =\left(h_{\alpha_{0}^{-1}}^{\varepsilon} \circ h_{\overline{\alpha_{0}}}^{\varepsilon}-i d\right) \circ h \frac{\varepsilon}{\alpha_{0}^{-1}}(t) \\
& =\left(h_{\alpha_{0}^{-1} \circ \overline{\alpha_{0}}}^{\varepsilon}-i d\right) \circ h \frac{\varepsilon}{\alpha_{0}^{-1}}(t) \\
& =\left(h_{\delta}^{\varepsilon}-i d\right) \circ h \frac{\varepsilon}{\alpha_{0}^{-1}}(t) .
\end{aligned}
$$

This, together with (11) and

$$
h \frac{\varepsilon}{\alpha_{0}^{-1}}(t)=t+O(\varepsilon), h_{\alpha_{0}^{-1}}^{\varepsilon}(t)=t+O(\varepsilon)
$$

implies

$$
\begin{aligned}
u & =t+O(\varepsilon) \\
v & =\frac{\varepsilon^{d}}{2 i}\left(M_{d}(t)+O(\varepsilon)\right)
\end{aligned}
$$

where, by abuse of notation, $O(\varepsilon)$ means a function analytic in $t, \varepsilon$, which vanishes identically for $\varepsilon=0$. This proves the identity $(9) . \triangle$

\section{Cyclicity of two-saddle cycles}

In this section we prove Theorem 1. Using the notations introduced in section 2, we suppose that the vector field $X_{0}$ has a two-saddle loop $\Gamma_{2}$ and an analytic first integral $f$ in a neighborhood of it. We suppose that $f$ has Morse critical points at the two saddle points $p_{1}, p_{2}$ of $X_{0}$. Consider the Dulac maps $d_{\varepsilon}^{1}, d_{\varepsilon}^{2}$ associated to the corresponding foliation, see fig. 4. Each map $d_{\varepsilon}^{i}$ is a composition of a "local" Dulac map (as in section 3) and two holomorphic holonomy maps. From this it follows that Lemma 2 applies to $d_{\varepsilon}^{i}, i=1,2$, too. We parameterize each cross-section by the restriction $z$ of $f$ on it. The function $d_{\varepsilon}^{i}, i=1,2$, is multivalued and has a critical point at $s_{i}(\varepsilon) \in \mathbb{R}, s_{i}(0)=0$. The functions $s_{i}$ are real analytic. We consider first the case $\varepsilon>0$ and we may suppose that $s_{i}(0)=0, s_{1}(\varepsilon)<s_{2}(\varepsilon)$ for all sufficiently small $\varepsilon$, (the case $\varepsilon<0$ is studied in the same way). Our aim is to bound the number of those zeros of the displacement map $d_{\varepsilon}^{1}-d_{\varepsilon}^{2}$ which are 


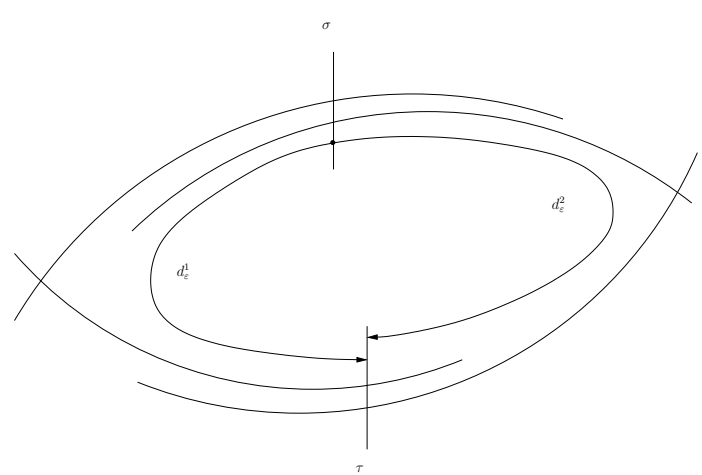

Figure 4: The Dulac maps $d_{\varepsilon}^{1}$ and $d_{\varepsilon}^{2}$

real, bigger than $s_{2}(\varepsilon)$ and tend to 0 as $\varepsilon$ tends to 0 . Note that these zeros correspond to the fixed points of the Poincare first return map $P_{\varepsilon}$. Indeed,

$$
d_{\varepsilon}^{1}-d_{\varepsilon}^{2}=d_{\varepsilon}^{2} \circ\left(\left(P_{\varepsilon}-i d\right), \text { where } d_{0}^{2}=i d .\right.
$$

We shall count the zeros of the displacement map in the larger complex domain $\mathcal{D}_{\varepsilon}$ of the universal covering of $\mathbb{C} \backslash\left\{s_{1}(\varepsilon)\right\}$ defined as follows. It is bounded by the circle

$$
S_{R}=\{z:|z|=R\},
$$

by the interval $\left[s_{1}(\varepsilon), s_{2}(\varepsilon)\right]$, and by the zero locus of the imaginary part of the Dulac map $d_{\varepsilon}^{1}$ for $\Re(z)<s_{1}(\varepsilon)$, as it is shown on fig.5. The numbers $\varepsilon, R$ are subject to certain conditions explained bellow. The zeros of an analytic function in a complex domain equal the increase of the argument of the function along the border of the domain, divided by $2 \pi$ (the argument principle). To bound the increase of the argument we shall count the number of the zeros of the imaginary part of the function, along the border of the domain.

Choose first the real numbers $\varepsilon_{0}, R>0$ as follows. Let

$$
d_{\varepsilon}^{1}(z)-d_{\varepsilon}^{2}(z)=\varepsilon^{d} M_{d}(z)+0\left(\varepsilon^{d+1}\right)
$$

and let $z^{\nu}(\log z)^{\mu}$ be the leading term of $M_{d}(z)$. Then, by $(13), \nu=\nu\left(P_{\varepsilon}\right)$ is the characteristic number of the Poincaré map $P_{\varepsilon}$ associated to $\Gamma_{2}$. We choose $R>0$ so small, that the increase of the argument of $M_{d}(z)$ along the circle $S_{R}$ is sufficiently close to the increase of the argument of $z^{\nu}(\log z) \mu$ 


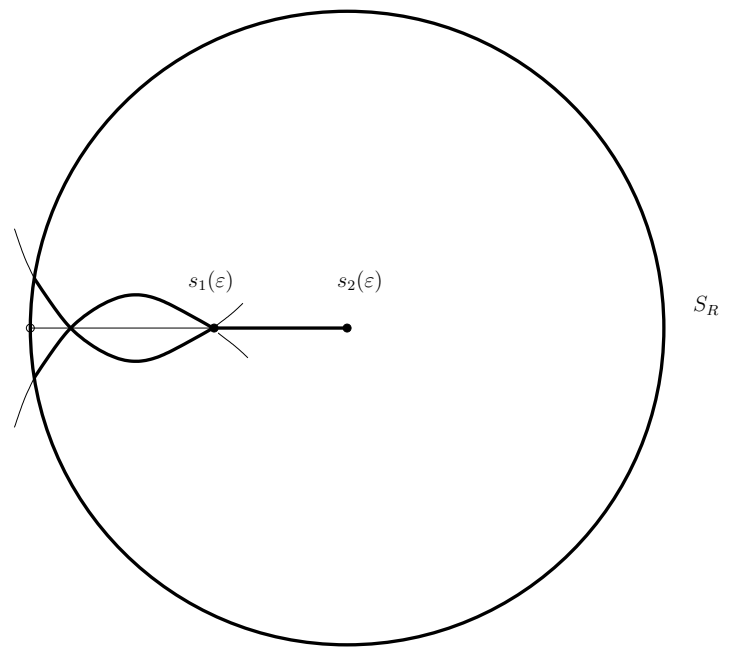

Figure 5: The domain $\mathcal{D}_{\varepsilon}$

along $S_{R}$. We fix $R$ and choose $\varepsilon_{0}>0$ so small with respect to $R$, that for all $\varepsilon,|\varepsilon|<\varepsilon_{0}$, the increase of the argument of $d_{\varepsilon}^{1}(z)-d_{\varepsilon}^{2}(z)$ along the circle $S_{R}$ is sufficiently close to the increase of the argument of $M_{d}(z)$ along $S_{R}$. This is indeed possible, according to Lemma 7. The conditions that we impose on $\varepsilon_{0}, \varepsilon$ and $R$ will be denoted (by abuse of notations) as follows

$$
1>R>>\varepsilon_{0}>\varepsilon>0 \text {. }
$$

To evaluate the increase of the argument of $d_{\varepsilon}^{1}(z)-d_{\varepsilon}^{2}(z)$ along the interval $\left[s_{1}(\varepsilon), s_{2}(\varepsilon)\right]$, we bound the zeros of its imaginary part which equals (along the interval $\left.\left[s_{1}(\varepsilon), s_{2}(\varepsilon)\right]\right)$ to the imaginary part of $-d_{\varepsilon}^{2}(z)$. In other words, we need to estimate the number of intersection points (counted with multiplicity) between the zero locus of the imaginary part of $d_{\varepsilon}^{2}(z)$ and $\left[s_{1}(\varepsilon), s_{2}(\varepsilon)\right]$. According to Lemma 2 this number of intersection points is bounded by the multiplicity of the zero at the origin of the Poincaré-Pontryagin function of the holonomy map $h_{\delta_{2}}^{\varepsilon}$. This multiplicity equals $\nu\left(h_{\delta_{2}}^{\varepsilon}\right)$.

Finally, we arrive at the most delicate point in the proof of Theorem 11: evaluate the increase of the argument of $d_{\varepsilon}^{1}(z)-d_{\varepsilon}^{2}(z)$ along the zero locus of the imaginary part of the Dulac map $d_{\varepsilon}^{1}$ for $\Re(z)<s_{1}(\varepsilon)$. For this purpose we bound the zeros of the imaginary part $\operatorname{Im}\left(d_{\varepsilon}^{1}(z)-d_{\varepsilon}^{2}(z)\right)$, along the zero locus of $\operatorname{Im} d_{\varepsilon}^{2}(z)$. Thus, we need to estimate the number of intersection 
points (counted with multiplicity) between the zero locus of the imaginary part of $d_{\varepsilon}^{2}(z)$, and the zero locus of the imaginary part of $d_{\varepsilon}^{1}(z)$.

Recall that to a Dulac map $d_{\varepsilon}^{i}$ we associated a family of vanishing loops $\delta_{i}(z)$ with orientation prescribed by 10$)$. With this convention, the orientation of the loops $\delta_{1}$ and $\delta_{2}$ do not agree : if $\gamma(t)$ is the family of periodic orbits of $\mathcal{F}_{0}$ for $t>0$, then the intersection indices of the homotopy classes of $\delta_{1}(t), \delta_{2}(t)$ with $\gamma(t)$ have opposite signs. In order to have the same convention as in the formulation of Theorem 1 we reverse the orientation of $\delta_{2}$. With this convention if

$$
h_{\delta_{i}}^{\varepsilon}: z \mapsto z+\varepsilon^{d^{i}} M_{d}^{i}(z)+\ldots
$$

then, by Lemma 2, the zero locus of the imaginary part of the holonomy maps $h_{\delta_{1}}^{\varepsilon}, h_{\delta_{2}}^{\varepsilon}$ is given by

$$
\begin{gathered}
\left\{z=u+i v: v=\frac{\varepsilon^{d^{1}}}{2 i} M_{d^{1}}(u)+\varepsilon^{d^{1}+1} R^{1}(u, \varepsilon), u<0\right\} \cap \mathcal{D} \\
\left\{z=u+i v: v=-\frac{\varepsilon^{d^{2}}}{2 i} M_{d^{2}}(u)+\varepsilon^{d^{2}+1} R^{2}(u, \varepsilon), u<0\right\} \cap \mathcal{D}
\end{gathered}
$$

respectively, where $R^{1}, R^{2}$ are appropriate analytic functions.

We conclude that the number of intersection points of the above analytic curves coincides with the multiplicity of the zero at the origin of either $M_{d^{1}}$ (if $d^{1}<d^{2}$ ), or $M_{d^{2}}$ (if $d^{2}<d^{1}$ ), or $M_{d^{1}}+M_{d^{2}}$ (if $d^{1}=d^{2}$ ). The number of intersection points equals therefore to the characteristic number $\nu\left(h_{\delta_{1}}^{\varepsilon} \circ h_{\delta_{2}}^{\varepsilon}\right)$.

Summing up the above information we get that the increase of the argument of $d_{\varepsilon}^{1}(z)-d_{\varepsilon}^{2}(z)$ along the boundary of the complex domain $\mathcal{D}_{\varepsilon}$ is not bigger than

$$
\nu\left(P_{\varepsilon}\right)+\nu\left(h_{\delta_{2}}^{\varepsilon}\right)+\nu\left(h_{\delta_{1}}^{\varepsilon} \circ h_{\delta_{2}}^{\varepsilon}\right)+2 .
$$

The above estimate can be slightly improved, by taking into consideration the fact that the imaginary part of $d_{\varepsilon}^{1}(z)-d_{\varepsilon}^{2}(z)$ vanishes at $s_{2}(\varepsilon)$. Theorem 1 is proved.

\section{A Proof of Theorem 2}

In this appendix we will prove Theorem 2 in the slightly more general context of multi-parameter analytic deformations. This will be used in Appendix B, Note that the zero-parameter case is well known [13, 19]. 


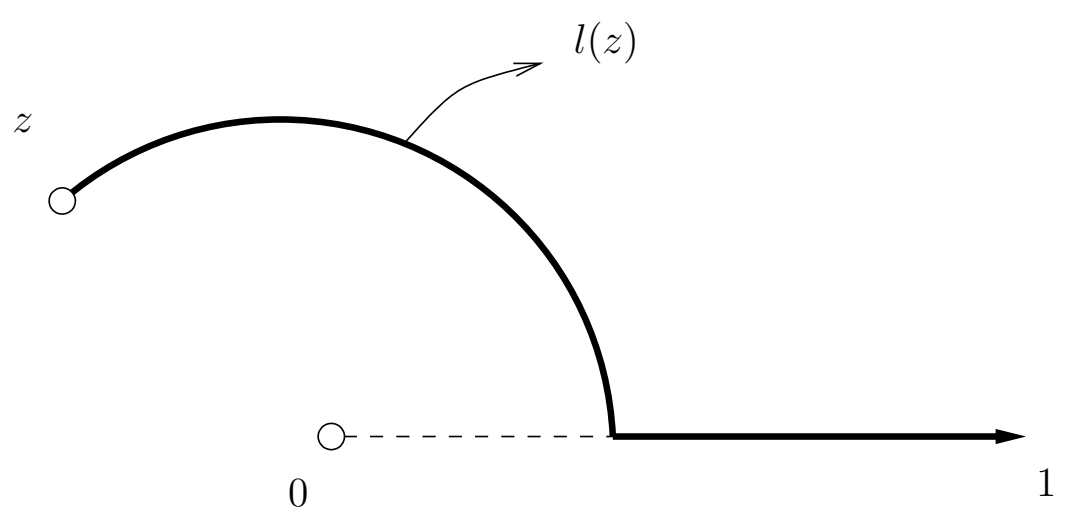

Figure 6: The path $l(z)$

Consider a $N$-parameter analytic family of analytic plane vector fields $X_{\lambda}, \lambda \in\left(\mathbb{R}^{N}, 0\right)$, such that $X_{0}$ has a hyperbolic singular point at the origin. It is known, since Briot and Bouquet, that $X_{0}$ has two transversal invariant analytic curves which can be supposed to coincide with the axes $x=0$ and $y=0$, that is to say

$$
X_{0}=\lambda_{1} x(1+\ldots) \frac{\partial}{\partial x}+\lambda_{2} y(1+\ldots) \frac{\partial}{\partial y}, \lambda_{1} \lambda_{2}<0 .
$$

The proof is as follows : a formal change of the variables first removes some (but not all) non-resonant terms, and then one verifies the convergency of the transformation, see [14, Appendice II]. Exactly the same proof applies, however, to the family $X_{\lambda}$. One can show in this way that $X_{\lambda}$ is analytically orbitally equivalent to the following (slightly improved) normal form

$$
x \frac{\partial}{\partial x}+y(r+x y \cdot a) \frac{\partial}{\partial y}
$$

where $r=r(\lambda), a=a(x, y, \lambda)$ are appropriate analytic functions in their arguments, $r(0)<0$, see [18, Appendice 1]. We shall suppose, without loss of generality, that there exists a constant $c>0$ such that $r, a$ are analytic in the complex domain

$$
\left.\mathcal{D}_{c}=\{x, y, \lambda):|x|<2,|y|<2,|\lambda|<c\right\} .
$$


After a further linear re-scaling of $x, y$, we may suppose that $|a(x, y, \lambda)|$ is so small in $\mathcal{D}_{c}$, that

$$
r(\lambda)+x y \cdot a(x, y, \lambda) \neq 0 .
$$

After this preparation, choose the cross-sections

$$
\sigma=\{y=1\}, \tau=\{x=1\}
$$

and consider the corresponding Dulac map

$$
\begin{aligned}
d_{\lambda}: \sigma & \rightarrow \tau \\
z & \mapsto d_{\lambda}(z) .
\end{aligned}
$$

To prove Theorem 2 we have to show that the constant $c>0$ can be chosen in such a way, that for every $\varphi_{0}>0$ there exists $0<z_{0}<1$, such that the Dulac map allows an analytic continuation in the sector

$$
\left\{z \in \mathbb{C}:|z|<z_{0},|\arg (z)|<\varphi_{0}\right\} \times\{\lambda:|\lambda|<c\} .
$$

The proof is similar to the proof of [19, Theorem 7], the only difference being the presence of the parameter $\lambda$. We shall construct a continuous family of paths $\gamma_{\lambda}(z)$ contained in the leaves of the foliation $\mathcal{F}_{\lambda}$ defined by the vector field $X_{\lambda}$ in $\mathbb{C}^{2}$. Each path $\gamma_{\lambda}(z)$ starts at the point $(x=z, y=1)$ and ends at the point $\left(x=1, y=d_{\lambda}(z)\right.$. The path $\gamma_{\lambda}(z)$ is constructed by lifting the path $l(z)$ contained in the $x$-plane $\{y=0\}$ and shown on fig.6. with respect to the projection

$$
\begin{aligned}
\pi: \mathbb{C}^{2} & \rightarrow \mathbb{C} \\
(x, y) & \mapsto x
\end{aligned}
$$

Indeed, the foliation $\mathcal{F}_{\lambda}$ is transversal to the projection $\pi$ except along the leaf $x=0$, provided that $r+x y . a \neq 0$. The resulting path path $\gamma_{\lambda}(z)$ is shown on fig. 7 .

To prove the existence of $\gamma_{\lambda}(z)$, consider the solution $y=y(x)$ associated to the vector field $X_{\lambda}$, with initial condition $y(z)=1$. We have to show that the solution $y=y(x)$ exists when $x$ is restricted to the path $l(z)$. The path $l(z)$ is composed by an arc and a segment. We consider them separately

- Along the arc

$$
x=|z| e^{i \varphi}, y=0,0<\varphi<\arg (z)
$$




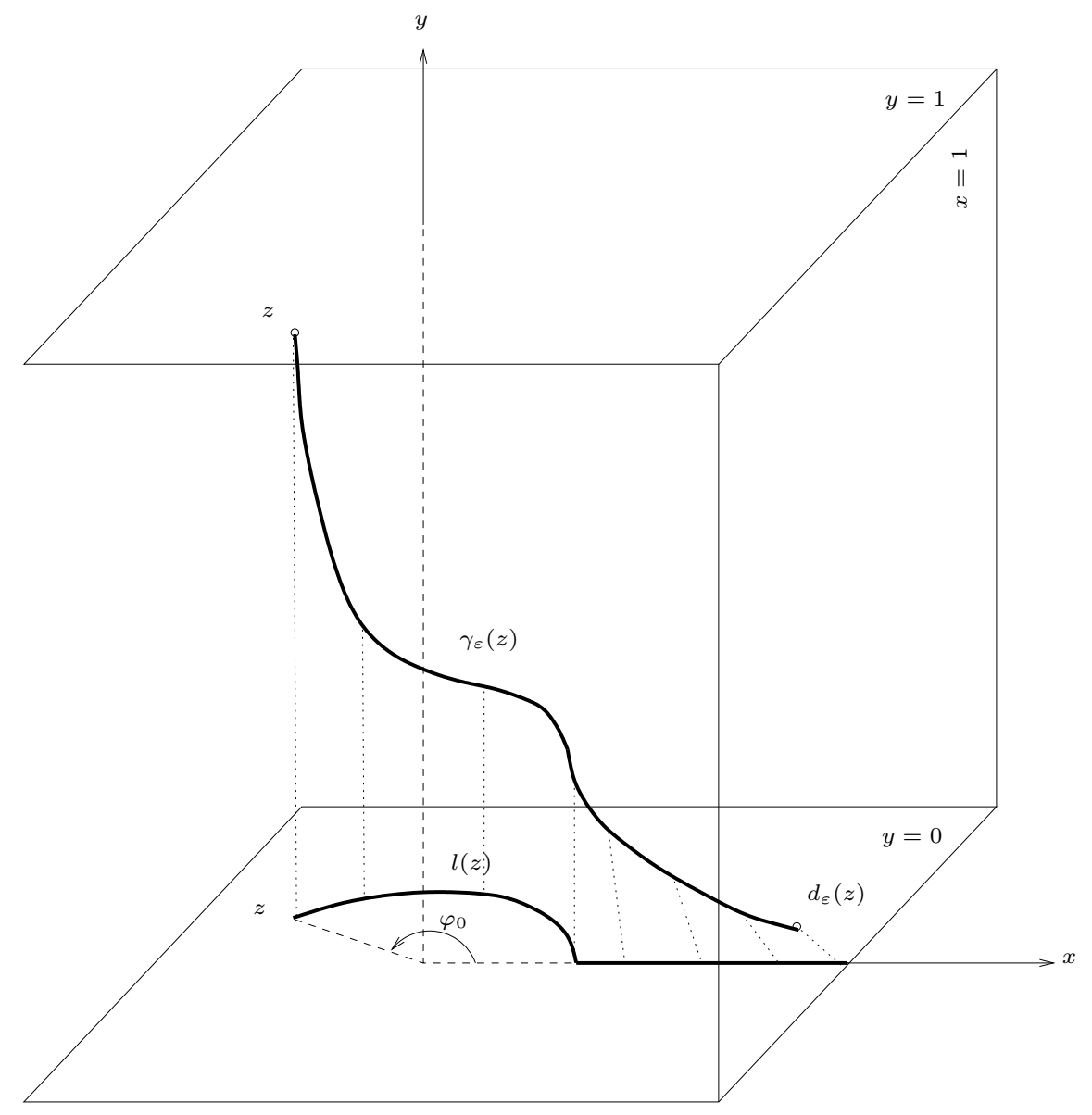

Figure 7: The path $\gamma_{\lambda}(z)$ 
parameterized by $\varphi$ we have

$$
\begin{aligned}
d y & =-y\left(r+|z| e^{i \varphi} y \cdot a\right) d \varphi \\
d|y| & =-|y| \cdot|z| \operatorname{Im}\left(e^{i \varphi} y . a\right) d \varphi .
\end{aligned}
$$

Therefore, if $|z|<z_{0}$ is sufficiently small, then $\left|y\left(|z| e^{i \varphi}\right)\right|<2$ when $0<\varphi<\arg (z)$

- Along the segment

$$
x \in[|z|, 1]
$$

we have similarly

$$
\begin{aligned}
x d y & =-y(r+x y \cdot a) d x \\
x d|y| & =-|y|(r+\Re(x y \cdot a)) d x .
\end{aligned}
$$

The derivative $\frac{d|y|}{d x}$ is therefore negative, the function $|y|(x)$ decreasing, so $|y|(x)<1$.

\section{B Multi-parameter deformations of Hamilto- nian two-saddle loops}

Consider, as in the preceding Appendix, a $N$-parameter analytic family of analytic plane vector fields $X_{\lambda}, \lambda \in\left(\mathbb{R}^{N}, 0\right)$. We suppose that $X_{0}$ has a Hamiltonian two-saddle loop $\Gamma_{2}$ bounding a period annulus. This case is easily reduced to the one-parameter case studied in the present paper by making use of a standard procedure based on the Hironaka desingularization theorem. In this Appendix we indicate the main steps.

\section{B.1 Principalization of the Bautin ideal}

In this section we follow [8, 20]. Let $z_{0} \in \sigma, z_{0} \notin \Gamma_{2}$ and consider the Poincaré map

$$
P_{\lambda}(z)=z+\sum_{i=1}^{\infty} a_{i}(\lambda)\left(z-z_{0}\right)^{i} .
$$


The Bautin ideal, associated to $P_{\lambda}$, is the ideal $\mathcal{I}=<a_{i}>$, generated by the germs of the analytic coefficients $a_{i}($.$) . It is Noetherian, so generated by$ a finite number of coefficients, and moreover does not depend on the choice of $z_{0}$ [19]. More generally, let $\{\gamma(z)\}$ be any continuous family of closed loops in the fibers of the foliation $\mathcal{F}_{0}$, intersecting the cross-section $\sigma$. For all sufficiently small $\lambda$ the (germ of) holonomy map

$$
h_{\gamma}^{\lambda}: \sigma \rightarrow \sigma
$$

is defined. In the same way, we associate to $h_{\gamma}^{\lambda}(z)$ a Bautin ideal, generated by the coefficients $a_{i}(\lambda)$ of the expansion of $h_{\gamma}^{\lambda}(z)$ with respect to $z-z_{0}$. As before it is Noetherian, and does not depend on the choice of $z_{0}$ (with the same proof).

We may assume, without any loss of generality, that the Bautin ideal is principal. For this we use a variant of Hironaka's desingularization theorem as follows.

Let $\varphi_{0}, \varphi_{1}, \ldots, \varphi_{p}$ be non-zero analytic functions on a smooth complex or real analytic variety $X$. The indeterminacy points of the rational map

$$
\varphi: X \rightarrow \mathbb{P}^{p}
$$

can be eliminated as follows [11, 2]

Theorem 3 (Hironaka desingularization) There exists a smooth analytic variety $\tilde{X}$ and a proper analytic map $\pi: \tilde{X} \rightarrow X$ such that the induced map $\tilde{\varphi}=\varphi \circ \pi$ is analytic.

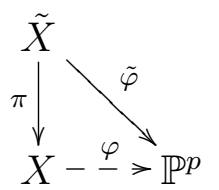

Let $\mathcal{O}_{X}$ be the sheaf of analytic functions on $X$ and consider the ideal sheaf $I \subset \mathcal{O}_{X}$ generated by $\varphi_{0}, \varphi_{1}, \ldots, \varphi_{p}$. The inverse image ideal sheaf of $I$ under the map $\pi: \tilde{X} \rightarrow X$ will be denoted $\pi^{*} I$. This is the ideal sheaf generated by the pull-backs of local sections of $I$. We note that $\pi^{*} I$ may differ from the usual sheaf-theoretic pull-back, also commonly denoted by $\pi^{*} I$. A simple consequence of Theorem 3 is the following

Corollary 1 The inverse image ideal sheaf $\pi^{*} I$ is principal. 
This is called the principalization of $I$. Indeed, as the induced map $\tilde{\varphi}$ is analytic, then for every $\tilde{\lambda} \in \tilde{X}$ there exists $j$, such that the functions $\tilde{\varphi}_{i} / \tilde{\varphi}_{j}$, $i=1,2, \ldots, p$, are analytic in a neighborhood of $\tilde{\lambda}$. Therefore there is a neighborhood $\tilde{U}$ of $\tilde{\lambda}$ such that $\left.\tilde{\varphi}_{j}\right|_{\tilde{U}}$ divides $\left.\tilde{\varphi}_{i}\right|_{\tilde{U}}$ in the ring of sections $\mathcal{O}_{\tilde{U}}$ of the sheaf $\mathcal{O}_{\tilde{X}}$, that is to say $I_{\tilde{U}}$ is generated by $\left.\tilde{\varphi}_{j}\right|_{\tilde{U}}$.

In our context $X=\sigma$ is the cross-section to the family of periodic orbits $\{\gamma(z)\}$ and $\varphi_{0}, \varphi_{1}, \ldots, \varphi_{p}$ are the germs of analytic functions which generate the Bautin ideal associated to the holonomy map $h_{\gamma}^{\lambda}$. To apply Theorem 3 we assume that $\sigma$ is a polydisc on which $\varphi_{i}$ are analytic, and the divisors $\left(\varphi_{i}\right)$ intersect transversally the boundary of $\sigma$. After applying the Hironaka's theorem, the origin 0 of $\sigma$ is replaced by a compact divisor $\pi^{-1}(0)$, along which the inverse image ideal sheaf $\pi^{*} I$ is principal.

Suppose now that we have a holonomy map $h_{\delta}^{\lambda}$ associated to another family of periodic orbits $\delta(t)$. Let $\psi_{0}, \psi_{1}, \ldots, \psi_{q}$ be generators of the corresponding Bautin ideal $J$. As before we assume that $\psi_{i}$ are analytic on $\sigma$, with divisors transversal to its boundary. Applying twice the Hironaka's theorem we get a new analytic variety smooth analytic variety $\tilde{X}$ and a proper analytic map $\pi: \tilde{X} \rightarrow X$ such that the induced maps $\tilde{\varphi}=\varphi \circ \pi$ and $\tilde{\psi}=\psi \circ \pi$ are analytic, see diagram (19).

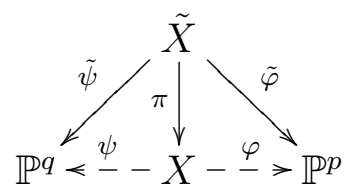

The inverse image ideal sheaf $\pi^{*} I$ and $\pi^{*} J$ are both principal along the compact divisor $\pi^{-1}(0)$.

\section{B.2 Multi-parameter version of Theorem 1}

We begin by formulating the multi-parameter version of Lemma 2, let $\{\delta(t)\}$ be the family of vanishing loops defined in section 3.3. In agreement with the preceding section, let us suppose that the Bautin ideal of the holonomy map $h_{\delta}^{\lambda}$ is principal. We have, therefore (compare to (11)

$$
\begin{aligned}
h_{\delta}^{\lambda}: \tau & \rightarrow \tau \\
z & \mapsto z+\tilde{\varphi}(\lambda)(\tilde{M}(z)+\tilde{R}(z, \lambda))
\end{aligned}
$$

where $\tilde{R}(.,$.$) is analytic, \tilde{R}(z, 0)=0$, and $\tilde{M}($.$) is the highest order Poincaré-$ Pontryagin function. 
Lemma 3 The zero locus $\mathcal{H}_{\varepsilon}$ of the imaginary part of the Dulac map is a smooth real-analytic curve of $\mathbb{R}^{2}=\mathbb{C}$ of the form

$$
\mathcal{H}_{\lambda}=\left\{z=u+i v: v=\frac{\tilde{\varphi}(\lambda)}{2 i}(\tilde{M}(u)+R(u, \lambda)), u<0\right\} \cap \mathcal{D}
$$

where $R(u, \lambda)$ is an analytic function, $R(u, 0)=0$.

The proof of the above Lemma is completely analogous to that of Lemma 2 and is therefore omitted.

In the proof of Theorem 1 we used four Bautin ideals associated to the holonomy maps

$$
P_{\lambda}=h_{\gamma}^{\lambda}, h_{\delta_{1}}^{\lambda}, h_{\delta_{2}}^{\lambda}, h_{\delta_{1}}^{\lambda} \circ h_{\delta_{2}}^{\lambda}
$$

where $\{\gamma(z)\}$ is the family of periodic orbits associated to the annulus, $\left\{\delta_{1}(z)\right\},\left\{\delta_{2}(z)\right\}$ are the vanishing families of loops associated to the saddle points. After an appropriate blow up $\pi$ we may suppose that the inverse image ideal sheafs of the corresponding four Bautin ideals are principal along the compact divisor $\pi^{-1}(0) \subset \tilde{X}$, see section B.1. Let $\tilde{\lambda}$ be a local variable on the smooth variety $\tilde{X}$. The cyclicity $\operatorname{Cycl}\left(\Gamma_{2},\left(\mathcal{F}_{\tilde{\lambda}}, \mathcal{F}_{\tilde{\lambda}_{0}}\right)\right)$ is the maximal number of limit cycles which $\mathcal{F}_{\tilde{\lambda}}$ can have in an arbitrarily small neighborhood of $\Gamma_{2}$, when $\tilde{\lambda}$ tends to $\tilde{\lambda}_{0}$. Denote also

$$
\operatorname{Cycl}\left(\Gamma_{2}, \mathcal{F}_{\lambda}\right)=\operatorname{Cycl}\left(\Gamma_{2},\left(\mathcal{F}_{\lambda}, \mathcal{F}_{0}\right)\right.
$$

Clearly

$$
\operatorname{Cycl}\left(\Gamma_{2}, \mathcal{F}_{\lambda}\right)=\sup _{\tilde{\lambda} \in \pi^{-1}(0)} \operatorname{Cycl}\left(\Gamma_{2},\left(\mathcal{F}_{\tilde{\lambda}}, \mathcal{F}_{\tilde{\lambda}_{0}}\right)\right)
$$

and because of the compactness of $\pi^{-1}(0)$, there exists $\tilde{\lambda}_{0} \in \pi^{-1}(0)$ such that

$$
\operatorname{Cycl}\left(\Gamma_{2}, \mathcal{F}_{\lambda}\right)=\operatorname{Cycl}\left(\Gamma_{2},\left(\mathcal{F}_{\tilde{\lambda}}, \mathcal{F}_{\tilde{\lambda}_{0}}\right)\right)
$$

The above considerations show that, without any harm, we may suppose that $\tilde{X}=\sigma, \lambda=\tilde{\lambda}, \tilde{\lambda}_{0}=0$, and the Bautin ideals associated to the holonomies (22) are principal. Consider the circle $S_{R}$ defined in (14). The Bautin ideal of the Poincaré map $P_{\lambda}$ coincides with the Bautin ideal of the displacement map $d_{\lambda}^{1}-d_{\lambda}^{2}$ and

$$
d_{\lambda}^{1}(z)-d_{\lambda}^{2}(z)=\varphi(\lambda)(M(z)+R(z, \lambda))
$$

where $\varphi$ is the generator of the Bautin ideal, $R$ is analytic, $R(z, 0)=0$, and $M(z)$ is the Poincaré-Pontryagin function. As before $M$ satisfies a Fuchs 
equation with a singularity at $z=0$. We choose $R$ so small, that the increase of the argument of $M(z)$ along $S_{R}, \arg (z)<\pi$ is close to the increase of the argument of the leading term of $M$. We note that if $z^{\nu}(\log (z))^{\mu}$ is the leading term of $M$, then

$$
\nu=\nu\left(P_{\lambda(\varepsilon)}\right)
$$

where $\varepsilon \rightarrow \lambda(\varepsilon)$ is a one-parameter deformation (a specialization), such that $\varphi(\lambda()) \neq$.0 . We fix $R$ and choose $\varepsilon_{0}>0$ so small, that for all $\lambda$, such that $|\lambda|<\varepsilon_{0}$, the increase of the argument of the displacement map $d_{\lambda}^{1}(z)-d_{\lambda}^{2}(z)$ along $S_{R}, \arg (z)<\pi$, is close to the increase of the argument of $M(z)$. By making use of Lemma 3 , the proof of Theorem 1 is completed as in section 4 . Note that the characteristic numbers which appear in the estimate of the cyclicity may be obtained as characteristic numbers corresponding to a oneparameter analytic deformation $\mathcal{F}_{\lambda(\varepsilon)}$ of $\mathcal{F}_{0}$

$$
\varepsilon \rightarrow \lambda(\varepsilon), \lambda(0)=0, \lim \tilde{\lambda}(\varepsilon)=\tilde{\lambda}_{0}
$$

provided that the generators of the Bautin ideals do not vanish identically along this deformation. Therefore, a multi-parameter version of Theorem 1 can be formulated as follows

Theorem 4 There exists a germ of analytic curve $\varepsilon \rightarrow \lambda(\varepsilon), \lambda(0)=0$ in the parameter space, such that

$$
\operatorname{Cycl}\left(\Gamma_{2}, \mathcal{F}_{\lambda}\right) \leq 1+\nu\left(P_{\lambda(\varepsilon)}\right)+\max \left\{\nu\left(h_{\delta_{1}}^{\lambda(\varepsilon)}\right), \nu\left(h_{\delta_{2}}^{\lambda(\varepsilon)}\right)\right\}+\nu\left(h_{\delta_{1}}^{\lambda(\varepsilon)} \circ h_{\delta_{2}}^{\lambda(\varepsilon)}\right) .
$$

\section{References}

[1] A. A. Andronov and E. A. Leontovič. On the generation of limit cycles from a loop of a separatrix and from the separatrix of the state of equilibrium of saddle-knot type. Mat. Sb. (N.S.), 48 (90):335-376, 1959.

[2] Edward Bierstone and Pierre D. Milman. Uniformization of analytic spaces. J. Amer. Math. Soc., 2(4):801-836, 1989.

[3] Magdalena Caubergh, Freddy Dumortier, and Robert Roussarie. Alien limit cycles in rigid unfoldings of a Hamiltonian 2-saddle cycle. Commun. Pure Appl. Anal., 6(1):1-21, 2007. 
[4] Freddy Dumortier and Robert Roussarie. Abelian integrals and limit cycles. J. Differential Equations, 227(1):116-165, 2006.

[5] J. P. Francoise. Successive derivatives of a first return map, application to the study of quadratic vector fields. Ergodic Theory Dynam. Systems, 16(1):87-96, 1996.

[6] J.-P. Francoise. The successive derivatives of the period function of a plane vector field. J. Differential Equations, 146(2):320-335, 1998.

[7] Lubomir Gavrilov. Higher order Poincaré-Pontryagin functions and iterated path integrals. Ann. Fac. Sci. Toulouse Math. (6), 14(4):663-682, 2005.

[8] Lubomir Gavrilov. Cyclicity of period annuli and principalization of Bautin ideals. Ergodic Theory Dynam. Systems, 28(5):1497-1507, 2008.

[9] Lubomir Gavrilov and Iliya D. Iliev. The displacement map associated to polynomial unfoldings of planar Hamiltonian vector fields. Amer. J. Math., 127(6):1153-1190, 2005.

[10] Lubomir Gavrilov and Dmitry Novikov. On the finite cyclicity of open period annuli. Duke Math. J., to appear, 2009.

[11] Heisuke Hironaka. Resolution of singularities of an algebraic variety over a field of characteristic zero. I, II. Ann. of Math. (2) 79 (1964), 109-203; ibid. (2), 79:205-326, 1964.

[12] Yu. Ilyashenko. Centennial history of Hilbert's 16th problem. Bull. Amer. Math. Soc. (N.S.), 39(3):301-354 (electronic), 2002.

[13] Frank Loray. Pseudo-groupe d'une singularité de feuilletage holomorphe en dimension deux. http: //hal . archives-ouvertes.fr/hal-00016434/en/.

[14] J.-F. Mattei and R. Moussu. Holonomie et intégrales premières. Ann. Sci. École Norm. Sup. (4), 13(4):469-523, 1980.

[15] G. S. Petrov. Nonoscillation of elliptic integrals. Funktsional. Anal. $i$ Prilozhen., 24(3):45-50, 96, 1990. 
[16] L.S. Pontryagin. Über Autoschwingungssysteme, die den Hamiltonschen nahe liegen. Phys. Z. Sowjetunion, 6:25-28, 1934.

[17] Robert Roussarie. On the number of limit cycles which appear by perturbation of separatrix loop of planar vector fields. Bol. Soc. Brasil. Mat., 17(2):67-101, 1986.

[18] Robert Roussarie. Cyclicité finie des lacets et des points cuspidaux. Nonlinearity, 2(1):73-117, 1989.

[19] Robert Roussarie. Bifurcation of planar vector fields and Hilbert's sixteenth problem, volume 164 of Progress in Mathematics. Birkhäuser Verlag, Basel, 1998.

[20] Robert Roussarie. Melnikov functions and Bautin ideal. Qual. Theory Dyn. Syst., 2(1):67-78, 2001. 